be detected in duodenal aspirates, intestinal biopsies, and impression smears of intestinal mucosa, but such high-powered investigations are really justifiable only in the investigation of long-continued diarrhoea and malabsorption states. ${ }^{1}$ The oval thick-walled cyst can be detected readily under the microscope, even in faeces of formed stools, and this simple examination is sufficient for routine diagnosis.

Infestation with $G$. lamblia may cause no symptoms. But if the infestation is heavy the patient usually suffers from diarrhoea and some upper abdominal discomfort. He may also have some general malaise and headache. Occasionally the abdominal pain is severe, and this may lead to a wrong diagnosis of appendicitis or other intra-abdominal condition. ${ }^{2}$ The diarrhoea tends to be persistent and can cause considerable loss of weight. Sometimes in children there are so many trophozoites coating the mucosa of the small intestine that its function is impaired and malabsorption results. ${ }^{13}$ The usual course of the illness, however, is a few days of diarrhoea and then a period of continuing loose stools for days or weeks. Man is the only host, and the disease spreads by the faecal-oral route. It can be a cause of endemic diarrhoea where hygiene is difficult to enforce, such as in institutions for infants and young children or in any areas where sanitation is absent or primitive. The infection can also be water-borne, and when this is the case sharp outbreaks of enteritis may occur. ${ }^{4}$ The incubation period is probably between one and four weeks, so that an outbreak is likely to occur late during a holiday or cruise or even after return home. In this way it differs from travellers' diarrhoea, which usually attacks tourists within a few days of their arriving at their holiday destination. ${ }^{4}$

Treatment with either metronidazole or mepacrine is effective. The adult dose of metronidazole is $200 \mathrm{mg}$ three times a day and of mepacrine $100 \mathrm{mg}$ three times a day for five to seven days. For children under 4 years old one-quarter of the adult dose is sufficient. Diarrhoea responds quickly, and in most cases cysts disappear from the faeces after one course of treatment. If they do not, a second course usually succeeds. The rare cases of malabsorption due to very heavy infestation in children require more thorough assessment in both diagnosis and tests of cure. ${ }^{1}$

1 Kamath, K. R., and Murugasu, R., Gastroenterology, 1974, 66, 16.

2 Geddes, A. M., British Medical fournal, 1973, 4, 236.

${ }^{3}$ Ament, M. E., and Rubin, C. E., Gastroenterology, 1972, 62, 216.

4 Walzer, P. D., Wolfe, M. S., and Shultz, M. G., Fournal of Infectious Diseases, 1971, 124, 235.

5 Thompson, R. G., Karandikar, D. S., and Leek, J., Lancet, 1974, 1, 615.

\section{Clinical Public Health Doctors}

In the rush to bring the N.H.S. reorganization into operation on 1 April many clinical public health doctors believe they have been forgotten. "Wait for the report of the Court Committee ${ }^{1}$ on child health services," they are told: but meanwhile little effort seems to have been made to provide them with any certainty about their future.

Perhaps the most obvious opportunity for integration of public health staff into the N.H.S. was in the field of child health. Since 1948 local authority child health services have made great progress, and in many authorities the school health service and the infant welfare service have been combined and have developed into a comprehensive screening and developmental assessment service for children from birth to school leaving age. These new services have been functioning at district level, based on child assessment centres provided either in the district general hospitals or in the community, and the centres have been given full support by specialist paediatricians. There was a great opportunity in the new N.H.S. to extend the integration of the local authcrity child health services and the hospital specialist paediatric services to provide a comprehensive district child health service. Further, as primary care services have developed and inany more general practitioners have moved into purpose-built accommodation this integration could perhaps have included the family doctor and his team.

Had such an integrated service been set up, the clinical public health doctors would have entered the district department of child health on the same terms as the hospital specialist staff. In this district department the work of the doctors would vary as it does now in paediatrics and local authority child health departments, some doctors being mainly concerned with sick children, some with handicapped children, some with developmental assessment and screening, and some with administration. Some would be of consultant status: others would not, but there would be a clear and attractive future for clinical and public health doctors as equal partners with the paediatricians. Instead the responsibility for child health has been included in the specialty of community medicine, and worse still the isolation of the school health service is to be perpetuated, since it is to be put in the charge of the area specialist in community medicine (child health). This area-based doctor is to be responsible for advising the education authority and will be in executive control of clinical public health doctors working in schools. The appointment is not consistent with the concept of a district department of child health and furthermore is seen by many experienced child health principal medical officers as the imposition of another doctor above them, thus degrading their own position.

It is understandable, therefore, that there is much unease amongst clinical public health doctors and morale is at a low ebb. The situation could still be saved by creating district specialists in community medicine (child health) who with the consultant paediatricians would manage a district child health service, the area specialist in community medicine (child health) being responsible for co-ordination, epidemiology, and medical education.

A similar approach could and should have been applied to other clinical public health doctors engaged in geriatrics, rehabilitation, mental health, and family planning, where there is also much uncertainty. No doubt a few of these doctors will obtain district community physician posts of the advertised area posts specialists in community medicine (social services). For the remainder there is at present nothing.

The uncertain future for clinical public health doctors is already affecting recruitment, and it is doubtful if the present high standards of service, particularly in the field of child health, can be maintained for long. The situation can be saved; but it is little help to say the Court Committee must report before anything can be done. Whatever the committee decides, true integration with the hospital services is essential if clinical standards are to be maintained.

\footnotetext{
1 British Medical fournal, 1973, 2, 789.
} 